$@$ ERSpublications

Benzodiazepines, regardless of half-life, are associated with adverse respiratory outcomes across COPD severity http://ow.ly/DgoFp

Nicholas T. Vozoris ${ }^{1,2}$ and Anne L. Stephenson ${ }^{1,2,3}$

${ }^{1}$ Division of Respirology, Dept of Medicine, St Michael's Hospital, Toronto, ON, Canada. ${ }^{2}$ Dept of Medicine, University of Toronto, Toronto, ON, Canada. ${ }^{3}$ Keenan Research Centre in the Li Ka Shing Knowledge Institute, St Michael's Hospital, Toronto, ON, Canada.

Correspondence: Nicholas T. Vozoris, Division of Respirology, Dept of Medicine, St Michael's Hospital, 30 Bond Street, Toronto, Ontario M5B 1W8, Canada. E-mail: nick.vozoris@utoronto.ca

Received: Sept 212014 | Accepted: Sept 222014

Conflict of interest: None declared.

\title{
References
}

1 Midgren B, Hansson L, Skeidsvoll H, et al. The effects of nitrazepam and flunitrazepam on oxygen desaturation during sleep in patients with stable hypoxemic nonhypercapnic COPD. Chest 1989; 95: 765-768.

2 Murciano D, Armengaud $\mathrm{MH}$, Cramer $\mathrm{PH}$, et al. Acute effects of zolpidem, triazolam and flunitrazepam on arterial blood gases and control of breathing in severe COPD. Eur Respir J 1993; 6: 625-629.

3 Vozoris NT, Fischer HD, Wang X, et al. Benzodiazepine drug use and adverse respiratory outcomes among older adults with COPD. Eur Respir J 2014; 44: 332-340.

4 Global Initiative for Chronic Obstructive Lung Disease. Global Strategy for the Diagnosis, Management, and Prevention of Chronic Obstructive Pulmonary Disease. www.goldcopd.org/uploads/users/files/GOLD_Report_2014_Jun11.pdf Date last updated: 2014. Date last accessed: September 2014.

5 Donaldson GC, Seemangal TA, Bhowmik A, et al. Relationship between exacerbation frequency and lung function decline in chronic obstructive pulmonary disease. Thorax 2002; 57: 847-852.

6 Connors AF Jr, Dawson NV, Thomas C, et al. Outcomes following acute exacerbation of severe chronic obstructive lung disease. The SUPPORT investigators (Study to Understand Prognoses and Preferences for Outcomes and Risks of Treatments). Am J Respir Crit Care Med 1996; 154: 959-967.

7 Hurst JR, Vestbo J, Anzueto A, et al. Susceptibility to exacerbation in chronic obstructive pulmonary disease. N Engl J Med 2010; 363: 1128-1138.

8 Klink ME, Dodge R, Quan SF. The relation of sleep complaints to respiratory symptoms in a general population. Chest 1994; 105: 151-154.

9 Ekström MP, Bornefalk-Hermansson A, Abernethy AP, et al. Safety of benzodiazepines and opioids in very severe respiratory disease: national prospective study. BMJ 2014; 348: g445.

10 Herings RM, Stricker BH, de Boer A, et al. Benzodiazepines and the risk of falling leading to femur fractures. Dosage more important than elimination half-life. Arch Intern Med 1995; 155: 1801-1807.

\section{HIV and multidrug-resistant tuberculosis: overlapping risk factors}

To the Editor:

Recent meta-analyses have indicated that, on average, new HIV-positive tuberculosis (TB) patients are at increased risk of multidrug-resistant (MDR)-TB compared with HIV-negative patients, while this risk is less clear for previously treated TB patients [1,2]. In a recent issue of the European Respiratory Journal, DEAN et al. [3] reported an association between HIV infection and MDR-TB disease based on aggregated data reported annually to the World Health Organization. Out of 24 countries where $\geqslant 75 \%$ of TB patients had a HIV test result and at least one HIV-positive MDR-TB case was reported, 11 showed a significant positive association between HIV and multidrug resistance among TB patients, mostly in countries with a high prevalence of multidrug resistance. One of these countries was Kazakhstan, which added data for 40975 out of the 104781 patients included in their report.

The authors pointed out that there are likely to be risk factors common to HIV-positive and MDR-TB patients that could not be explored in their analysis. They refer to a study from Moldova, where detailed analysis of 2007-2010 surveillance data showed that the positive association observed among new patients remained after adjustment for potential confounders [4]. We would like to refer to a similar analysis 

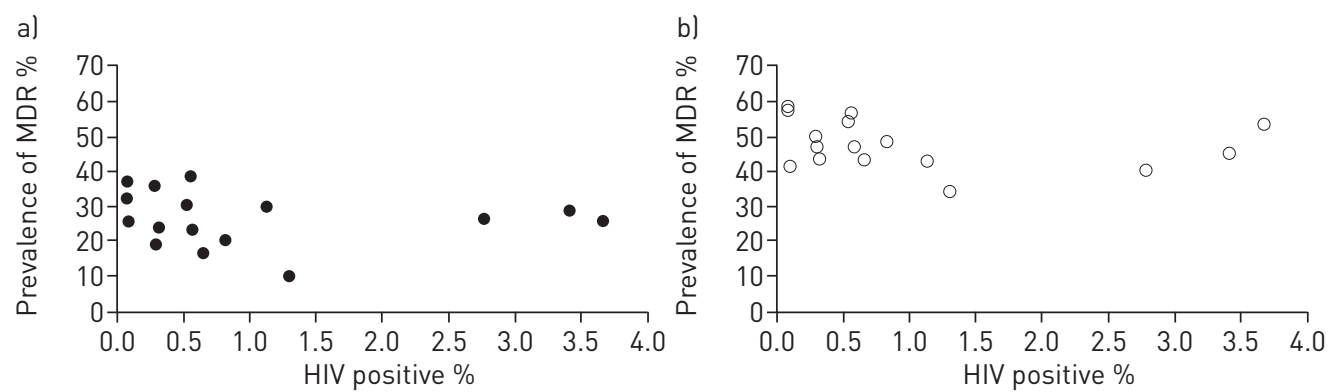

FIGURE 1 Prevalence of multidrug resistance (MDR) and HIV among a) new and b) previously treated tuberculosis patients in Kazakhstan, 2007-2011.

performed in Kazakhstan [5]. Using routine data from the electronic, national TB register from 20072011, we assessed patient characteristics associated with MDR-TB and/or HIV. In Kazakhstan, all patients are tested for HIV and TB drug resistance. HIV test results were available for $97 \%$ of TB patients and drug susceptibility test results for $93 \%$ of culture-positive patients. The proportion of TB patients with HIV increased from $0.6 \%$ in 2007 to $1.5 \%$ in 2011 . The proportion of TB patients with multidrug resistance was high but did not change over time; on average, it was $36.1 \%$ for new and retreatment patients combined. In a crude analysis, among all 50589 TB patients with HIV and drug susceptibility test results available, we observed a $20 \%$ higher prevalence of MDR-TB among HIV-positive patients than among HIV-negative patients (OR 1.2, 95\% CI 1.02-1.4). Our results revealed that risk factors among TB patients for HIV and multidrug resistance were largely overlapping; in a univariate analysis, both included male sex, young adult age, urban residence, a history of incarceration, homelessness and drug abuse. Moreover, after adjustment for these overlapping risk factors in addition to demographics and treatment history, this association disappeared completely (adjusted OR 1.0, 95\% CI 0.86-1.2). In addition, at the regional level, no clear association was observed between the prevalence of multidrug resistance and HIV (fig. 1). On average, $0.6 \%$ of the TB patients were infected both with HIV and MDR-TB Mycobacterium tuberculosis, but this risk varied greatly. TB patients at highest risk of being infected both with HIV and MDR-TB M. tuberculosis were those using drugs (12.5\%) and those with a history of imprisonment (3.4\%). These findings indicate that in Kazakhstan, the dual epidemic of HIV and MDR-TB is converging in specific socially vulnerable groups, and that enhanced efforts are necessary to provide (access to) diagnosis, TB/ HIV treatment and care to these groups. This is important as both HIV and MDR-TB put patients at increased risk of treatment failure and mortality, and their combination is even more dangerous $[6,7]$. Determinants of HIV and multidrug resistance are at least partly setting-specific and whether there is an independent association between them also may be situational. Therefore, it is important that more countries assess whether specific determinants may explain an apparent crude association between HIV and MDR-TB. This will require additional data collection beyond that available in routine surveillance registers in most settings.

-

@ERSpublications

Overlapping risk factors may explain observed association between HIV and drug resistance in TB patients http://ow.ly/DdRGT

Susan van den Hof ${ }^{1,2}$, Aigul Tursynbayeva ${ }^{3}$, Tleukhan Abildaev ${ }^{4}$, Malik Adenov $^{4}$, Svetlana Pak $^{3}$ and Shakhimurat Ismailov ${ }^{5}$

${ }^{1}$ KNCV Tuberculosis Foundation, The Hague, The Netherlands. ${ }^{2}$ Academic Medical Center and Amsterdam Institute of Global Health and Development, Amsterdam, The Netherlands. ${ }^{3} \mathrm{KNCV}$ Tuberculosis Foundation, Representative Office for Central Asia, Almaty, Kazakhstan. ${ }^{4}$ National Center of Tuberculosis Problem, Almaty, Kazakhstan. ${ }^{5}$ Global Fund against AIDS, Tuberculosis and Malaria - Tuberculosis Grant Project Implementation Unit, Almaty, Kazakhstan.

Correspondence: Susan van den Hof, KNCV Tuberculosis Foundation, PO Box 416, 2501 CC, The Hague, The Netherlands. Email: susan.vandenhof@kncvtbc.org

Received: July 182014 | Accepted: Aug 122014

Conflict of interest: None declared.

\section{References}

1 Mesfin YM, Hailemariam D, Biadglign S, et al. Association between HIV/AIDS and multi-drug resistance tuberculosis: a systematic review and meta-analysis. PLoS One 2014; 9: e82235.

2 Suchindran S, Brouwer ES, Van Rie A. Is HIV infection a risk factor for multi-drug resistant tuberculosis? A systematic review. PLoS One 2009; 4: e5561. 
3 Dean AS, Zignol M, Falzon D, et al. HIV and multidrug-resistant tuberculosis: overlapping epidemics. Eur Respir J 2014; 44: 251-254.

4 Jenkins HE, Plesca V, Ciobanu A, et al. Assessing spatial heterogeneity of multidrug-resistant tuberculosis in a high-burden country. Eur Respir J 2013; 42: 1291-1301.

5 van den Hof S, Tursynbayeva A, Abildaev T, et al. Converging risk factors but no association between HIV infection and multidrug-resistant tuberculosis in Kazakhstan. Int J Tuberc Lung Dis 2013; 17: 526-531.

6 Wells CD, Cegielski JP, Nelson LJ, et al. HIV infection and multidrug-resistant tuberculosis: the perfect storm. J Infect Dis 2007; 196: Suppl. 1, S86-S107.

7 Podlekareva DN, Panteleev AM, Grint D, et al. Short- and long-term mortality and causes of death in HIV/ tuberculosis patients in Europe. Eur Respir J 2014; 43: 166-177.

\section{The role of therapeutic drug monitoring in individualised drug dosage and exposure measurement in tuberculosis and HIV co-infection}

To the Editor:

We read with interest the paper by Esposito et al. [1] reporting a difficult-to-treat extensively drug-resistant tuberculosis (TB) case. TB treatment in HIV-positive individuals can also be particularly challenging. An HIV-positive 26-year-old male showing excessive weight loss (body mass index $17.5 \mathrm{~kg} \cdot \mathrm{m}^{-2}$ ) and clinical deterioration was admitted to the Beatrixoord Tuberculosis Centre (University Medical Center Groningen, Haren, the Netherlands) for the treatment of pulmonary TB. Mycobacterium tuberculosis isolated from sputum appeared susceptible to all first-line drugs tested. The patient received rifampicin (RIF) $(600 \mathrm{mg})$, isoniazid (300 mg), pyrazinamide (1500 mg) and ethambutol (1200 $\mathrm{mg})$ under directly observed therapy. He started with emtricitabine $(200 \mathrm{mg})$, tenofovir $(245 \mathrm{mg})$ and raltegravir $(800$ mg twice daily) 2 weeks later as combination antiretroviral therapy. Therapeutic drug monitoring (TDM) was performed to evaluate the extent of the effect of RIF on raltegravir. Multiple blood samples were drawn over a period of $12 \mathrm{~h}$ to evaluate RIF exposure. Although raltegravir concentrations were adequate, strikingly low RIF concentrations were measured [2]. Unfortunately, RIF concentrations were not measured in an earlier stage of the treatment. Newly obtained plasma samples confirmed the low RIF concentration. To detect a potential decreased absorption, the same dosage of RIF was administered intravenously, which resulted in an acceptable RIF exposure (fig. 1). Drug-drug interactions influencing the absorption of RIF were not expected based on the concomitantly administered medication. The patient had no gastro-intestinal complaints and the faeces showed no presence of Giardia lamblia, Entamoeba histolytica and Cryptosporidium spp. Between the start of the TB treatment and the TDM day he had gained $11.2 \mathrm{~kg}$. No obvious signs were present that could account for the remarkably low drug exposure after oral administration. However, despite the decreased bioavailability of RIF, the patient responded well to therapy. Because a decreased bioavailability was noticed in a late phase of treatment and considering the positive treatment outcome, it was decided not to change treatment. The patient has remained clinically well and free of relapse 2 years after completion of TB treatment.

Drug exposure of first-line anti-TB drugs has gained renewed interest after the hollow-fibre infection model showed that the effectiveness of these drugs is driven by the ratio of area under the curve (AUC) of concentration-time to minimum inhibitory concentration (MIC) [3]. In addition, evidence is accumulating that subtherapeutic concentrations may contribute to acquired drug resistance and treatment failure [4]. A reduction of anti-TB drug exposure, in particular RIF, in HIV patients was reported earlier [5], but not in all studies [6]. Enteropathy caused by parasitic infections or by HIV itself (enterocyte apoptosis) and diarrhoea can often explain the reduced drug absorption in HIV patients. In our patient, the AUC from time zero to $24 \mathrm{~h}$ after dosing (AUC0-24) was $2.43 \mathrm{mg} \cdot \mathrm{h} \cdot \mathrm{L}^{-1}$ after oral administration and $29.92 \mathrm{mg} \cdot \mathrm{h} \cdot \mathrm{L}^{-1}$ after i.v. administration, resulting in an estimated bioavailability of $8.12 \%$. For this specific case we speculate that the exposures of concomitantly administered anti-TB drugs were sufficient and 